Projektbereich B

Discussion Paper No. B-259

\title{
Risk-Minimizing Hedging Strategies under Restricted Information
}

\author{
by \\ Martin Schweizer *)
}

November 1993

*) Financial support by Deutsche Forschungsgemeinschaft, Sonderforschungsbereich 303 at the University of Bonn, is gratefully acknowledged.

(Mathematical Finance 4 (1994), 327-342)

This version: 04.05.1995 


\title{
Risk-Minimizing Hedging Strategies under Restricted Information
}

\author{
Martin Schweizer* \\ Universität Göttingen \\ Institut für Mathematische Stochastik \\ Lotzestraße 13 \\ D-37083 Göttingen \\ Germany
}

\begin{abstract}
We construct risk-minimizing hedging strategies in the case where there are restrictions on the available information. The underlying price process is a $d$ dimensional $\mathbb{F}$-martingale, and strategies $\varphi=(\vartheta, \eta)$ are constrained to have $\vartheta \mathbb{G}$-predictable and $\eta \mathbb{G}^{\prime}$-adapted for filtrations $\mathbb{G} \subseteq \mathbb{G}^{\prime} \subseteq \mathbb{F}$. We show that there exists a unique $\left(\mathbb{G}, \mathbb{G}^{\prime}\right)$-risk-minimizing strategy for every contingent claim $H \in \mathcal{L}^{2}\left(\mathcal{G}_{T}^{\prime}, P\right)$ and provide an explicit expression in terms of $\mathbb{G}_{-}$predictable dual projections. Previous results of Föllmer/Sondermann (1986) and Di Masi/Platen/Runggaldier (1993) are recovered as special cases. Examples include a Black-Scholes model with delayed information and a jump process model with discrete observations.
\end{abstract}

Key words: option hedging, risk-minimizing strategies, restricted information, predictable dual projection

1991 Mathematics Subject Classification: 60G44, 60H05, 90A09

* Financial support by Deutsche Forschungsgemeinschaft, Sonderforschungsbereich 303 at the University of Bonn, is gratefully acknowledged. 


\section{Introduction}

This paper provides a construction of risk-minimizing hedging strategies in the case where there are restrictions on the available information. We consider a model with one riskless asset with (discounted) price 1 and $d$ risky assets whose (discounted) prices are given by an $\mathbb{R}^{d}$-valued locally square-integrable local martingale $X$. The process $X$ is adapted to a (large) filtration $\mathbb{F}$ and so the results of Föllmer/Sondermann (1986) imply that there exists a unique $\mathbb{F}$-risk-minimizing hedging strategy $\varphi=(\vartheta, \eta)$ for every contingent claim $H \in \mathcal{L}^{2}\left(\mathcal{F}_{T}, P\right)$. The processes $\vartheta$ and $\eta$ are $\mathbb{F}$-predictable and $\mathbb{F}$-adapted, respectively; $\vartheta$ is given by the integrand of $X$ in the Galtchouk-Kunita-Watanabe decomposition of $H$ with respect to $\mathbb{F}$, while $\eta$ is determined by the requirement that the value process $V(\varphi)=\vartheta^{*} X+\eta$ should coincide with the $\mathbb{F}$-martingale $E[H \mid \mathbb{F}]$.

Here we are interested in situations where the hedger has less information than $\mathbb{F}$. We therefore consider two smaller filtrations $\mathbb{G} \subseteq \mathbb{G}^{\prime} \subseteq \mathbb{F}$ and look for $\left(\mathbb{G}, \mathbb{G}^{\prime}\right)$-risk-minimizing

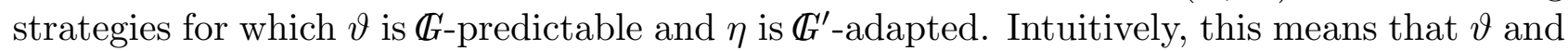
$\eta$ have to be constructed using only the information available in $\mathbb{G}$ and $\mathbb{G}^{\prime}$, respectively. This question is of considerable interest from a practical point of view. We may for instance think of a situation where stock prices can only be observed at discrete time instants and hedging strategies have to be based on these observations. This can be modelled in our framework by choosing filtrations $\mathbb{G}, \mathbb{G}^{\prime}$ which are piecewise constant. A particular example of this type was studied by Di Masi/Platen/Runggaldier (1993) and provided the motivation for the more general analysis presented here.

The paper starts in section 1 with a brief review of the results of Föllmer/Sondermann (1986) on risk-minimization in the case $\mathbb{G}=\mathbb{G}^{\prime}=\mathbb{F}$ of unrestricted information. In addition to providing definitions and results, we have also tried to explain and motivate the concept of risk-minimizing hedging strategies since this may be new to some readers. In section 2 , we solve the general case of restricted information. Since $X$ is usually neither $\mathbb{G}$ - nor $\mathbb{G}^{\prime}$ adapted, there is typically no Galtchouk-Kunita-Watanabe decomposition, and so we need a different approach. In a first step, we show that a strategy $\varphi=(\vartheta, \eta)$ with $V_{T}(\varphi)=H$ $P$-a.s. is $\left(\mathbb{G}, \mathbb{G}^{\prime}\right)$-risk-minimizing if and only if the $\mathbb{G}^{\prime}$-optional projection of the cost process $C(\varphi)=V(\varphi)-\int \vartheta d X$ is a $\mathbb{G}^{\prime}$-martingale and if $\vartheta$ solves

$$
\text { Minimize } E\left[\left(H-\int_{0}^{T} \gamma_{u} d X_{u}\right)^{2}\right] \text { over all } \mathbb{G} \text {-predictable } \gamma \in L^{2}(X) \text {. }
$$

The definition of $L^{2}(X)$ is given in section 1. The above condition on $C(\varphi)$ can be used to determine $\eta$ from $\vartheta$. Then we show that $(0.1)$ has a unique solution $\vartheta^{H}$ for every $H \in \mathcal{L}^{2}\left(\mathcal{G}_{T}^{\prime}, P\right)$, and we give an explicit expression for $\vartheta^{H}$ in terms of the Radon-Nikodym derivative of two $\mathbb{G}$-predictable dual projections. Section 3 concludes the paper with several special cases and examples. These include the results of Föllmer/Sondermann (1986) and Di Masi/Platen/Runggaldier (1993), an example with delayed information and a jump process example with discrete observations.

\section{A review of risk-minimization for local martingales}

Let $(\Omega, \mathcal{F}, P)$ be a probability space with a filtration $\mathbb{F}=\left(\mathcal{F}_{t}\right)_{0 \leq t \leq T}$ satisfying the usual conditions of right-continuity and completeness, where $T>0$ is a fixed and finite time 
horizon. Without special mention, all stochastic processes will be defined for $t \in[0, T]$. Let $X$ be an $\mathbb{R}^{d}$-valued local $\mathbb{F}$-martingale in $\mathcal{M}_{\text {loc }}^{2}(P, \mathbb{F})$ and denote by $\left\langle X^{i}\right\rangle$ the sharp bracket process associated to $X^{i}-X_{0}^{i}$ with respect to $P$ and $\mathbb{F}$ for $i=1, \ldots, d$. We can and shall choose a version of $X$ such that each $X^{i}$ is right-continuous with left limits (RCLL, for short). Fix an increasing $\mathbb{F}$-predictable RCLL process $B$ null at 0 such that $\left\langle X^{i}\right\rangle \ll B$ for $i=1, \ldots, d$ and define the $\mathbb{F}$-predictable matrix-valued process $\sigma$ by

$$
\sigma_{t}^{i j}:=\frac{d\left\langle X^{i}, X^{j}\right\rangle_{t}}{d B_{t}} \quad \text { for } i, j=1, \ldots, d .
$$

Definition. The space $\Theta(\mathbb{F}):=L^{2}(X, \mathbb{F})$ consists of all $\mathbb{R}^{d}$-valued $\mathbb{F}$-predictable processes $\vartheta$ satisfying

$$
E\left[\int_{0}^{T} \vartheta_{s}^{*} \sigma_{s} \vartheta_{s} d B_{s}\right]<\infty
$$

* denotes transposition.

Definition. An $\mathbb{F}$-strategy $\varphi$ is a pair of processes $\varphi=(\vartheta, \eta)$ with $\vartheta \in \Theta(\mathbb{F})$ and $\eta$ $\mathbb{F}$-adapted and such that the value process $V_{t}(\varphi):=\vartheta_{t}^{*} X_{t}+\eta_{t}$ is RCLL and satisfies

$$
\sup _{0 \leq t \leq T}\left|V_{t}(\varphi)\right| \in \mathcal{L}^{2}
$$

The (cumulative) cost process of $\varphi$ is given by

$$
C_{t}(\varphi):=V_{t}(\varphi)-\int_{0}^{t} \vartheta_{u} d X_{u}
$$

Finally, the $\mathbb{F}$-risk process of $\varphi$ is defined by

$$
R_{t}(\varphi):=E\left[\left(C_{T}(\varphi)-C_{t}(\varphi)\right)^{2} \mid \mathcal{F}_{t}\right]
$$

For interpretation and motivation, we refer to Harrison/Pliska (1981), Föllmer/Sondermann (1986) and the remark following (1.2).

Definition. An $\mathbb{F}$-strategy $\varphi=(\vartheta, \eta)$ is called $\mathbb{F}$-risk-minimizing if for any $t \in[0, T]$ and any $\mathbb{F}$-strategy $\widetilde{\varphi}=(\widetilde{\vartheta}, \widetilde{\eta})$ satisfying

$$
\begin{aligned}
V_{T}(\widetilde{\varphi}) & =V_{T}(\varphi) & P \text {-a.s. } \\
\widetilde{\vartheta}_{s} & =\vartheta_{s} & \text { for } s \leq t, \\
\widetilde{\eta}_{s} & =\eta_{s} & \text { for } s<t
\end{aligned}
$$

we have

$$
R_{t}(\widetilde{\varphi}) \geq R_{t}(\varphi) \quad P \text {-a.s. }
$$

Note that this definition is slightly different from the original one due to Föllmer/Sondermann (1986); the modification in the second equation in (1.1) first appeared in Schweizer (1988). For comparison purposes below, we provide the following equivalence result: 
Lemma 1.1. An $\mathbb{F}$-strategy $\varphi$ is $\mathbb{F}$-risk-minimizing if and only if

$$
R_{t}(\psi) \geq R_{t}(\varphi) \quad P \text {-a.s. }
$$

for every $t \in[0, T]$ and every $\mathbb{F}$-strategy $\psi$ such that $V_{T}(\psi)=V_{T}(\varphi) P$-a.s.

Proof. Since the "if" part is obvious, fix $t \in[0, T]$ and an $\mathbb{F}$-strategy $\psi=(\zeta, \chi)$ with $V_{T}(\psi)=V_{T}(\varphi) P$-a.s. If we define $\widetilde{\varphi}=(\widetilde{\vartheta}, \widetilde{\eta})$ by setting

$$
\widetilde{\vartheta}:=\vartheta I_{[0, t]}+\zeta I_{(t, T]}
$$

and

$$
V(\widetilde{\varphi}):=V(\varphi) I_{[0, t)}+V(\psi) I_{[t, T]},
$$

where $\varphi=(\vartheta, \eta)$, then $\widetilde{\varphi}$ clearly satisfies (1.1). But

$$
C_{T}(\widetilde{\varphi})-C_{t}(\widetilde{\varphi})=C_{T}(\psi)-C_{t}(\psi)
$$

and so we also obtain the "only if" part.

q.e.d.

Definition. Given a random variable $H \in \mathcal{L}^{2}\left(\mathcal{F}_{T}, P\right)$, an $\mathbb{F}$-strategy $\varphi$ is called $H$-admissible if $V_{T}(\varphi)=H$ P-a.s.

With the preceding terminology, the problem of risk-minimization with unrestricted information is now

$$
\text { Given } H \in \mathcal{L}^{2}\left(\mathcal{F}_{T}, P\right) \text {, find an } H \text {-admissible } \mathbb{F} \text {-risk-minimizing } \mathbb{F} \text {-strategy. }
$$

Remark. For those readers not already familiar with the concept of risk-minimization, some words of explanation may be useful here. We interpret $X$ as the discounted price process of a bundle of $d$ risky assets, and we assume that there also exists a riskless asset whose discounted price is 1 at all times. An $\mathbb{F}$-strategy then describes a dynamic portfolio strategy: $\vartheta_{t}^{i}$ is the number of shares of asset $i$ to be held at time $t$, while $\eta_{t}$ is the amount invested in the riskless asset. $V_{t}(\varphi)$ is then clearly the value at time $t$ of such a portfolio, and $C_{t}(\varphi)$ gives the cumulative costs up to time $t$ as current value minus total gains from trade. The random variable $H$ is called a contingent claim and models a random loss suffered at time $T$. For instance, the writer of a European call option on asset $i$ with strike $K$ would have to deal with $H=\left(X_{T}^{i}-K\right)^{+}$. In order to hedge against $H$, one can use the existing assets to construct an $H$-admissible $\mathbb{F}$-strategy; this corresponds to duplicating a contingent claim by means of a suitable hedging portfolio. If this can be done in a self-financing way, we obtain a unique arbitrage-free price for $H$ and there is no need for further optimization; see Harrison/Pliska (1981). In an incomplete market, however, duplication will in general not be possible using only self-financing strategies, and so the cost process will not be constant, but fluctuate randomly over time. Hence we need an optimality criterion to compare different strategies.

Intuitively, risk-minimization should be viewed as a procedure of sequential varianceminimization. To motivate this approach, consider any $H$-admissible strategy $\varphi$. Since $X$ is a martingale, the total cost

$$
C_{T}(\varphi)=H-\int_{0}^{T} \vartheta_{s} d X_{s}
$$


has expectation $E[H]$ independently of $\varphi$, and so it seems natural to try and minimize the variance $\operatorname{Var}\left[C_{T}(\varphi)\right]$. But it turns out that this is not sufficient to determine the entire strategy $\varphi$; only the component $\vartheta$ and the initial investment $\eta_{0}$ can be deduced. To obtain a unique optimal strategy $\varphi=(\vartheta, \eta)$, one has to impose more stringent conditions, and this is achieved by the preceding definitions. For a more detailed account, we refer to Föllmer/Sondermann (1986).

The solution of (1.2) is due to Föllmer/Sondermann (1986). For its formulation, we recall the well-known Galtchouk-Kunita-Watanabe decomposition: Every $H \in \mathcal{L}^{2}\left(\mathcal{F}_{T}, P\right)$ can be uniquely written as

$$
H=H_{0}+\int_{0}^{T} \xi_{u}^{H} d X_{u}+L_{T}^{H} \quad P \text {-a.s. }
$$

where $H_{0} \in \mathbb{R}, \xi^{H} \in \Theta(\mathbb{F})$ and $L^{H} \in \mathcal{M}^{2}(P, \mathbb{F})$ satisfies $E\left[L_{0}^{H}\right]=0$ and is strongly orthogonal to $X^{i}$ for each $i$. In fact, (1.3) is obtained by projecting the $\mathbb{F}$-martingale

$$
V_{t}^{H}:=E\left[H \mid \mathcal{F}_{t}\right]
$$

on the stable subspace of $\mathcal{M}^{2}(P, \mathbb{F})$ generated by $X^{1}-X_{0}^{1}, \ldots, X^{d}-X_{0}^{d}$. As a special case of Theorem 2.5 below, we then have the basic result of Föllmer/Sondermann (1986):

Proposition 1.2. For every $H \in \mathcal{L}^{2}\left(\mathcal{F}_{T}, P\right)$, there exists a unique $H$-admissible $\mathbb{I}$-riskminimizing $\mathbb{F}$-strategy given by

$$
\varphi_{t}^{H, I F}=\left(\xi_{t}^{H}, V_{t}^{H}-\left(\xi_{t}^{H}\right)^{*} X_{t}\right) .
$$

Remark. Due to (1.3), $V^{H}$ is given by

$$
V_{t}^{H}=H_{0}+\int_{0}^{t} \xi_{u}^{H} d X_{u}+L_{t}^{H}
$$

This implies for each $i$ that

$$
\left\langle V^{H}, X^{i}\right\rangle_{t}=\sum_{j=1}^{d} \int_{0}^{t}\left(\xi_{u}^{H}\right)^{j} d\left\langle X^{j}, X^{i}\right\rangle_{u}=\int_{0}^{t}\left(\sigma_{u} \xi_{u}^{H}\right)^{i} d B_{u}
$$

The optimal strategy is therefore given by

$$
\xi_{t}^{H}=\sigma_{t}^{\operatorname{inv}} \frac{d\left\langle V^{H}, X\right\rangle_{t}}{d B_{t}}
$$

where $A^{\text {inv }}$ denotes the pseudo-inverse of a matrix $A$. For $d=1$, we can choose $B \equiv\langle X\rangle$, $\sigma \equiv 1$, and so (1.4) simplifies to

$$
\xi_{t}^{H}=\frac{d\left\langle V^{H}, X\right\rangle_{t}}{d\langle X\rangle_{t}}
$$




\section{Risk-minimization under restricted information}

Suppose now that the hedger does not have at his disposal the full information represented by $\mathbb{F}$; his strategy must be constructed from less information. To describe this mathematically, we introduce two additional filtrations $\mathbb{G}=\left(\mathcal{G}_{t}\right)_{0 \leq t \leq T}$ and $\mathbb{G}^{\prime}=\left(\mathcal{G}_{t}^{\prime}\right)_{0 \leq t \leq T}$ satisfying the usual conditions and such that

$$
\mathcal{G}_{t} \subseteq \mathcal{G}_{t}^{\prime} \subseteq \mathcal{F}_{t} \quad \text { for every } t \in[0, T]
$$

Moreover, we shall assume that

$$
X_{T} \text { is } \mathcal{G}_{T}^{\prime} \text {-measurable. }
$$

Remarks. 1) Why consider two filtrations $\mathbb{G} \subseteq \mathbb{G}^{\prime}$ ? For one thing, this lets us see more clearly the structure of the measurability conditions which have to be imposed. More importantly, however, we shall want $\vartheta$ to be based on $\mathbb{G}, \eta$ on $\mathbb{G}^{\prime}$, and it seems natural that there should be more restrictions, hence less information, about trading in stocks than in a riskless asset. The simplest example would be discrete-time interventions on the stock market where

$$
\mathcal{G}_{t}=\mathcal{F}_{t_{k}} \quad \text { for } t \in\left[t_{k}, t_{k+1}\right)
$$

and $0=t_{0}<t_{1}<\ldots<t_{n}=T$. At the same time, we might well have full observations of $X$, and so we could take $\mathbb{G}^{\prime}=\mathbb{F}$ to model continuous rebalancing of the investment in the riskless asset.

2) Note that apart from (2.1), we impose no measurability conditions on $X$ with respect to $\mathbb{G}$ or $\mathbb{G}^{\prime}$. In particular, $X$ need not be $\mathbb{G}_{\text {- }}$ or $\mathbb{G}^{\prime}$-adapted and thus will not be a $\mathbb{G}^{-}$or $\mathbb{G}^{\prime}$ martingale in general. The restriction (2.1) is perfectly natural: working with $H$-admissible strategies means that we hedge on a cash settlement basis, and so we should of course be allowed to know the terminal value $V_{T}(\varphi)$ of our portfolio at time $T$. Choosing buy-andhold strategies of the form $\vartheta^{(i)} \equiv(0, \ldots, 0,1,0, \ldots, 0)^{*}$ and $\eta \equiv X_{0}^{i}$ for $i=1, \ldots, d$ then yields (2.1). Since we shall want to hedge $\mathcal{G}_{T}^{\prime}$-measurable claims, (2.1) is also an immediate requirement if we want at least to be able to hedge all call options on $X$.

Definition. The space $\Theta(\mathbb{G})$ consists of all $\mathbb{R}^{d}$-valued $\mathbb{G}$-predictable processes $\vartheta$ satisfying

$$
E\left[\int_{0}^{T} \vartheta_{s}^{*} \sigma_{s} \vartheta_{s} d B_{s}\right]<\infty .
$$

If we denote by $\mathcal{P}(\mathbb{G})$ the space of all $\mathbb{R}^{d}$-valued $\mathbb{G}$-predictable processes, then clearly $\Theta(\mathbb{G})=$ $\Theta(\mathbb{F}) \cap \mathcal{P}(\mathbb{G})$.

Definition. A $\left(\mathbb{G}, \mathbb{G}^{\prime}\right)$-strategy or simply strategy $\varphi=(\vartheta, \eta)$ is an $\mathbb{F}$-strategy such that $\vartheta \in \Theta(\mathbb{G})$ and $\eta$ is $\mathbb{G}^{\prime}$-adapted. The $\left(\mathbb{G}, \mathbb{G}^{\prime}\right)$-risk process of $\varphi$ is then defined by

$$
R_{t}^{G_{t}}(\varphi):=E\left[\left(C_{T}(\varphi)-C_{t}(\varphi)\right)^{2} \mid \mathcal{G}_{t}\right]=E\left[R_{t}(\varphi) \mid \mathcal{G}_{t}\right]
$$

Definition. A strategy $\varphi=(\vartheta, \eta)$ is called $\left(\mathbb{G}, \mathbb{G}^{\prime}\right)$-risk-minimizing if for any $t \in[0, T]$ and any strategy $\widetilde{\varphi}=(\widetilde{\vartheta}, \widetilde{\eta})$ satisfying $(1.1)$, we have

$$
R_{t}^{G_{t}}(\widetilde{\varphi}) \geq R_{t}^{G_{t}}(\varphi) \quad P \text {-a.s. }
$$


Remark. Di Masi/Platen/Runggaldier (1993) define $\left(\mathbb{G}, \mathbb{G}^{\prime}\right)$-risk-minimality in a less general framework by requiring that

$$
R_{t}^{G_{t}}(\widetilde{\varphi}) \geq R_{t}^{G_{t}}(\varphi) \quad P \text {-a.s. }
$$

for every $\left(\mathscr{G}, \mathbb{G}^{\prime}\right)$-strategy $\widetilde{\varphi}$ and every $t \in[0, T]$ such that $X_{t}$ is $\mathcal{G}_{t}^{\prime}$-measurable. The last condition on $t$ is rather restrictive, in particular if $\mathbb{G}^{\prime}$ is relatively small compared to $\mathbb{F}$. If $X$ is $\mathbb{G}^{\prime}$-adapted, Lemma 1.1 shows that the two definitions are equivalent. In general, however, we feel that our definition is the more natural extension of the original idea of Föllmer/Sondermann (1986).

Definition. Given $H \in \mathcal{L}^{2}\left(\mathcal{G}_{T}^{\prime}, P\right)$, a strategy $\varphi$ is called $H$-admissible if $V_{T}(\varphi)=H$-a.s.

With the preceding notations, we can now formulate the problem of risk-minimization under restricted information as

(2.3) Given $H \in \mathcal{L}^{2}\left(\mathcal{G}_{T}^{\prime}, P\right)$, find an $H$-admissible $\left(\mathbb{G}, \mathbb{G}^{\prime}\right)$-risk-minimizing $\left(\mathbb{G}\right.$, $\left.\mathbb{G}^{\prime}\right)$-strategy.

As explained above, (2.3) can be viewed as a problem of sequential variance-minimization, with additional measurability requirements on $\vartheta$ and $\eta$. Since $X$ is usually not $\mathbb{G}^{\prime}$-adapted, there is no Galtchouk-Kunita-Watanabe decomposition, and so we have to develop a different approach. We begin our analysis of (2.3) with a technical but important improvement lemma; the basic idea for this goes back to Schweizer (1988).

Lemma 2.1. For any strategy $\varphi=(\vartheta, \eta)$ and any $t \in[0, T]$, there exists a strategy $\widehat{\varphi}=(\vartheta, \widehat{\eta})$ satisfying

$$
\begin{gathered}
V_{T}(\widehat{\varphi})=V_{T}(\varphi) \quad P \text {-a.s. } \\
\widehat{\eta}_{s}=\eta_{s} \quad \text { for } s<t, \\
E\left[C_{T}(\widehat{\varphi})-C_{s}(\widehat{\varphi}) \mid \mathcal{G}_{s}^{\prime}\right]=0 \quad P \text {-a.s. for } s \geq t
\end{gathered}
$$

and

$$
R_{s}^{G_{i}}(\widehat{\varphi}) \leq R_{s}^{G_{s}}(\varphi) \quad P \text {-a.s. for } s \geq t .
$$

$\widehat{\eta}$ can be chosen to satisfy

$$
\widehat{\eta}_{s}=E\left[V_{T}(\varphi)-\vartheta_{s}^{*} X_{s} \mid \mathcal{G}_{s}^{\prime}\right] \quad \text { for } s \geq t .
$$

Proof. Fix $t$ and $\varphi$. Denote by $J$ the $\mathbb{G}^{\prime}$-optional projection of $V(\varphi)$, by $K$ an RCLL version of the $\mathbb{G}^{\prime}$-martingale $K_{t}:=E\left[V_{T}(\varphi) \mid \mathcal{G}_{t}^{\prime}\right]$ and define $\hat{\eta}$ by setting

$$
V_{s}(\widehat{\varphi}):=V_{s}(\varphi)+\left(K_{s}-J_{s}\right) I_{[t, T]}(s) .
$$

Then Theorem VI.47 of Dellacherie/Meyer (1982) implies that $\widehat{\varphi}$ is indeed a strategy, (2.5) is obvious and (2.4) follows from (2.1). By the definition of $\widehat{\varphi}$, we have for $s \geq t$

$$
\begin{aligned}
C_{T}(\widehat{\varphi})-C_{s}(\widehat{\varphi}) & =V_{T}(\widehat{\varphi})-V_{s}(\widehat{\varphi})-\int_{s}^{T} \vartheta_{u} d X_{u} \\
& =V_{T}(\varphi)-V_{s}(\varphi)-\int_{s}^{T} \vartheta_{u} d X_{u}+J_{s}-K_{s} \\
& =C_{T}(\varphi)-C_{s}(\varphi)+J_{s}-K_{s},
\end{aligned}
$$


hence (2.6) and (equivalently) (2.7). Finally, (2.8) implies for $s \geq t$ that

$$
R_{s}^{G_{t}}(\varphi)=R_{s}^{G_{F}}(\widehat{\varphi})+E\left[\left(J_{s}-K_{s}\right)^{2} \mid \mathcal{G}_{s}\right]
$$

by $(2.6)$.

q.e.d.

Definition. For any strategy $\varphi$, denote by $C^{\prime}(\varphi)$ the $\mathbb{G}^{\prime}$-optional projection of the cost process $C(\varphi)$. We call $\varphi$ weakly $\left(\mathbb{G}, \mathbb{G}^{\prime}\right)$-mean-self-financing if $C^{\prime}(\varphi)$ is a $\mathbb{G}^{\prime}$-martingale. This implies that

$$
E\left[C_{T}(\varphi)-C_{t}(\varphi) \mid \mathcal{G}_{t}^{\prime}\right]=0 \quad P \text {-a.s. for all } t \in[0, T]
$$

or equivalently

$$
\eta_{t}=E\left[V_{T}(\varphi)-\vartheta_{t}^{*} X_{t} \mid \mathcal{G}_{t}^{\prime}\right] \quad P \text {-a.s. for all } t \in[0, T]
$$

Corollary 2.2. If a strategy $\varphi$ is $\left(\mathbb{G}, \mathbb{G}^{\prime}\right)$-risk-minimizing, it is weakly $\left(\mathbb{G}, \mathbb{G}^{\prime}\right)$-mean-self-financing.

Proof. Construct $\widehat{\varphi}$ as in Lemma 2.1 with $t=0$. Since $\varphi$ is $\left(\mathbb{G}, \mathbb{G}^{\prime}\right)$-risk-minimizing, (2.9) implies that $J$ and $K$ are versions of each other, hence indistinguishable, since both are RCLL, and so we get

$$
C(\varphi)=V(\varphi)-\int \vartheta d X=V(\varphi)-J+K-\int \vartheta d X
$$

But $K$ and $\int \vartheta d X$ are $\mathbb{G}^{\prime}$ - and $\mathbb{F}$-martingales, respectively, and $J$ is the $\mathbb{G}^{\prime}$-optional projection of $V(\varphi)$; hence we conclude that $C^{\prime}(\varphi)$ is a $\mathbb{G}^{\prime}$-martingale.

q.e.d.

In order to solve (2.3), we now introduce the additional optimization problem

$$
\text { Given } H \in \mathcal{L}^{2}\left(\mathcal{G}_{T}^{\prime}, P\right) \text {, minimize } E\left[\left(H-\int_{0}^{T} \gamma_{u} d X_{u}\right)^{2}\right] \text { over all } \gamma \in \Theta\left(\mathbb{G}_{T}\right) \text {. }
$$

The next result provides the link between (2.3) and (2.11).

Proposition 2.3. Fix $H \in \mathcal{L}^{2}\left(\mathcal{G}_{T}^{\prime}, P\right)$. Then an $H$-admissible strategy $\varphi=(\vartheta, \eta)$ is $\left(\mathbb{G}\right.$, $\left.\mathbb{G}^{\prime}\right)$ risk-minimizing if and only if $\varphi$ is weakly $\left(\mathbb{G}, \mathbb{G}^{\prime}\right)$-mean-self-financing and $\vartheta$ solves (2.11).

Proof. 1) By the projection theorem, a process $\xi \in \Theta(\mathbb{G})$ solves (2.11) if and only if

$$
E\left[\left(H-\int_{0}^{T} \xi_{u} d X_{u}\right) \int_{0}^{T} \gamma_{u} d X_{u}\right]=0 \quad \text { for every } \gamma \in \Theta(\mathbb{G}) .
$$

By (1.3), this is equivalent to

$$
E\left[\int_{0}^{T}\left(\xi_{u}^{H}-\xi_{u}\right) d X_{u} \int_{0}^{T} \gamma_{u} d X_{u}\right]=0 \quad \text { for every } \gamma \in \Theta(\mathbb{G})
$$


or also to

$$
E\left[\int_{t}^{T}\left(\xi_{u}^{H}-\xi_{u}\right) d X_{u} \int_{t}^{T} \gamma_{u} d X_{u} \mid \mathcal{G}_{t}\right]=0 \quad P \text {-a.s. }
$$

for every $t \in[0, T]$ and every $\gamma \in \Theta(\mathbb{G})$. Finally, this is also equivalent to saying that

$$
E\left[\left(\int_{t}^{T} \gamma_{u} d X_{u}\right)^{2}-2 \int_{t}^{T}\left(\xi_{u}^{H}-\xi_{u}\right) d X_{u} \int_{t}^{T} \gamma_{u} d X_{u} \mid \mathcal{G}_{t}\right] \geq 0 \quad P \text {-a.s. }
$$

for every $t \in[0, T]$ and every $\gamma \in \Theta(\mathbb{G})$.

2) If $\varphi=(\vartheta, \eta)$ is $H$-admissible and $\left(\mathscr{G}, \mathbb{G}^{\prime}\right)$-risk-minimizing, then $\varphi$ is weakly $\left(\mathbb{G}, \mathbb{G}^{\prime}\right)$ mean-self-financing by Corollary 2.2. Fix $t \in[0, T]$ and consider any strategy $\widetilde{\varphi}=(\widetilde{\vartheta}, \widetilde{\eta})$ satisfying (1.1). If we construct $\widehat{\widetilde{\varphi}}$ by Lemma 2.1 , then $\widehat{\widetilde{\varphi}}$ also satisfies (1.1). Moreover, (2.7), (1.1) and (2.10) imply that $\widehat{\widetilde{\eta}}_{t}=\eta_{t}$, hence $V_{t}(\widehat{\widetilde{\varphi}})=V_{t}(\varphi)$ by (1.1) and therefore

$$
C_{T}(\varphi)-C_{t}(\varphi)=V_{T}(\varphi)-V_{t}(\varphi)-\int_{t}^{T} \vartheta_{u} d X_{u}=C_{T}(\widehat{\widetilde{\varphi}})-C_{t}(\widehat{\widetilde{\varphi}})+\int_{t}^{T}\left(\widetilde{\vartheta}_{u}-\vartheta_{u}\right) d X_{u}
$$

again by $(1.1)$. Since $\varphi$ is $\left(\mathbb{G}, \mathbb{G}^{\prime}\right)$-risk-minimizing, we deduce that

$$
\begin{aligned}
0 & \leq R_{t}^{G_{t}}(\widehat{\widetilde{\varphi}})-R_{t}^{G_{t}}(\varphi) \\
& =E\left[\left(\int_{t}^{T}\left(\widetilde{\vartheta}_{u}-\vartheta_{u}\right) d X_{u}\right)^{2}-2\left(C_{T}(\varphi)-C_{t}(\varphi)\right) \int_{t}^{T}\left(\widetilde{\vartheta}_{u}-\vartheta_{u}\right) d X_{u} \mid \mathcal{G}_{t}\right] \\
& =E\left[\left(\int_{t}^{T}\left(\widetilde{\vartheta}_{u}-\vartheta_{u}\right) d X_{u}\right)^{2}-2 \int_{t}^{T}\left(\xi_{u}^{H}-\vartheta_{u}\right) d X_{u} \int_{t}^{T}\left(\widetilde{\vartheta}_{u}-\vartheta_{u}\right) d X_{u} \mid \mathcal{G}_{t}\right]
\end{aligned}
$$

by (1.3). Choosing $\widetilde{\vartheta}:=\vartheta+\gamma I_{(t, T]}$ yields (2.12) and thus shows that $\vartheta$ solves (2.11).

3) Conversely, suppose that $\varphi=(\vartheta, \eta)$ is weakly $\left(\mathbb{G}, \mathbb{G}^{\prime}\right)$-mean-self-financing and $\vartheta$ solves (2.11). Fix $t \in[0, T]$ and consider any strategy $\widetilde{\varphi}=(\widetilde{\vartheta}, \widetilde{\eta})$ satisfying (1.1). The same construction as in step 2) leads again to (2.13), so choosing $\gamma:=\widetilde{\vartheta}-\vartheta$ in (2.12) yields as in step 2)

$$
0 \leq R_{t}^{G_{i}}(\widehat{\widetilde{\varphi}})-R_{t}^{G_{t}}(\varphi) \leq R_{t}^{G_{i}}(\widetilde{\varphi})-R_{t}^{G_{i}}(\varphi)
$$

by $(2.13)$ and Lemma 2.1 , and this shows that $\varphi$ is $\left(\mathbb{G}, \mathbb{G}^{\prime}\right)$-risk-minimizing.

q.e.d.

To prove existence and uniqueness of a solution to (2.11), we need a bit of notation. For any locally integrable RCLL process $A$ of finite variation, we denote by $A^{p, G}$ the $\mathbb{G}_{\text {- }}$ predictable dual projection of $A$. If $A \ll \widetilde{A}$, then also $A^{p, G_{r}} \ll \widetilde{A}^{p, G_{F}} ;$ this allows us to define the $\mathbb{G}$-predictable matrix-valued process $\varrho$ by setting

$$
\varrho_{t}^{i j}:=\frac{d\left(\int \sigma^{i j} d B\right)_{t}^{p, G}}{d B_{t}^{p, G}} \quad \text { for } i, j=1, \ldots, d .
$$


The next result is related to Stricker's lemma; see Proposition 1.2 of Stricker (1990).

Lemma 2.4. For every $H \in \mathcal{L}^{2}\left(\mathcal{G}_{T}^{\prime}, P\right)$, (2.11) has a unique solution $\vartheta^{H}$ in $\Theta(G)$.

Proof. Since the stochastic integral $\int_{0}^{T} \gamma_{u} d X_{u}$ is an isometry from $L^{2}(X, \mathbb{F})$ to $\mathcal{L}^{2}\left(\mathcal{F}_{T}, P\right)$, it is enough to show that $\Theta(\mathbb{G})$ is a closed subspace of $L^{2}(X, \mathbb{F})$. This is not clear from (2.2) alone, since $\sigma$ and $B$ are not necessarily $\mathbb{G}$-predictable. But using (2.14), we can rewrite (2.2) for $\gamma \in \Theta(\mathbb{G})$ as

$$
E\left[\int_{0}^{T} \gamma_{s}^{*} \sigma_{s} \gamma_{s} d B_{s}\right]=\sum_{i, j=1}^{d} E\left[\int_{0}^{T} \gamma_{s}^{i} \gamma_{s}^{j} d\left(\int \sigma^{i j} d B\right)_{s}\right]=E\left[\int_{0}^{T} \gamma_{s}^{*} \varrho_{s} \gamma_{s} d B_{s}^{p, G}\right],
$$

and since $\varrho$ and $B^{p, \mathbb{G}_{r}}$ are both $\mathbb{G}_{\boldsymbol{T}}$-predictable, it is obvious that $\Theta(\mathbb{G})$ is closed in $L^{2}(X, \mathbb{F})$.

q.e.d.

Here is now the central result on risk-minimization under restricted information:

Theorem 2.5. For any $H \in \mathcal{L}^{2}\left(\mathcal{G}_{T}^{\prime}, P\right)$, there exists a unique $H$-admissible $\left(\mathbb{G}, \mathbb{G}^{\prime}\right)$-risk-minimizing strategy $\varphi^{H}=\left(\vartheta^{H}, \eta^{H}\right)$. It is given by

$$
\vartheta_{t}^{H}=\varrho_{t}^{\operatorname{inv}} \frac{d\left(\int \sigma \xi^{H} d B\right)_{t}^{p, G}}{d B_{t}^{p, G_{r}}}
$$

and

$$
\eta_{t}^{H}=E\left[H-\left(\vartheta_{t}^{H}\right)^{*} X_{t} \mid \mathcal{G}_{t}^{\prime}\right]
$$

Proof. Existence and uniqueness of $\varphi^{H}$ follow from Lemma 2.4 and Proposition 2.3, and (2.16) follows from (2.10). By part 1) of the proof of Proposition 2.3, $\vartheta^{H}$ is determined by the condition that

$$
0=E\left[\left(H-\int_{0}^{T} \vartheta_{u}^{H} d X_{u}\right) \int_{0}^{T} \gamma_{u} d X_{u}\right]=E\left[\int_{0}^{T}\left(\xi_{u}^{H}-\vartheta_{u}^{H}\right) d X_{u} \int_{0}^{T} \gamma_{u} d X_{u}\right]
$$

for every $\gamma \in \Theta(\mathbb{G})$, where we have used (1.3). By the definition of the $\mathbb{G}$-predictable dual projection and (2.14), (2.17) can be rewritten as

$$
\begin{aligned}
0 & =E\left[\int_{0}^{T} \gamma_{u}^{*} \sigma_{u} \xi_{u}^{H} d B_{u}\right]-E\left[\int_{0}^{T} \gamma_{u}^{*} \sigma_{u} \vartheta_{u}^{H} d B_{u}\right] \\
& =E\left[\int_{0}^{T} \gamma_{u}^{*} d\left(\int \sigma \xi^{H} d B\right)_{u}\right]-\sum_{i, j=1}^{d} E\left[\int_{0}^{T} \gamma_{u}^{i}\left(\vartheta_{u}^{H}\right)^{j} d\left(\int \sigma^{i j} d B\right)_{u}\right] \\
& =E\left[\int_{0}^{T} \gamma_{u}^{*} d\left(\int \sigma \xi^{H} d B\right)_{u}^{p, G}\right]-E\left[\int_{0}^{T} \gamma_{u}^{*} \varrho_{u} \vartheta_{u}^{H} d B_{u}^{p, G}\right] .
\end{aligned}
$$


Since this holds for all $\gamma \in \Theta(\mathbb{G})$, we conclude that

$$
\varrho \vartheta^{H}=\frac{d\left(\int \sigma \xi^{H} d B\right)^{p, G_{r}}}{d B^{p, G_{r}}},
$$

hence (2.15).

q.e.d.

\section{Special cases and examples}

\subsection{The case of unrestricted information}

If we choose $\mathbb{G}=\mathbb{G}^{\prime}=\mathbb{F}$, we recover the "classical" case treated by Föllmer/Sondermann (1986). Since $\sigma, \xi^{H}$ and $B$ are then all $\mathbb{G}$-predictable, (2.15) simplifies to

$$
\vartheta_{t}^{H}=\sigma_{t}^{\mathrm{inv}} \frac{d\left(\int \sigma \xi^{H} d B\right)_{t}}{d B_{t}}=\xi_{t}^{H}
$$

and (2.16) reduces to

$$
\eta_{t}^{H}=V_{t}^{H}-\left(\vartheta_{t}^{H}\right)^{*} X_{t}=V_{t}^{H}-\left(\xi_{t}^{H}\right)^{*} X_{t} .
$$

Thus we obtain Proposition 1.2 as a special case of Theorem 2.5.

\subsection{The case where $B$ is $\mathbb{G}$-predictable}

Now suppose that the process $B$ is $\mathbb{G}$-predictable. Then we obtain

$$
\left(\int \gamma d B\right)^{p, G_{r}}=\int^{p, G_{r}} \gamma d B
$$

for every $\gamma$, where ${ }^{p, G_{F}} \gamma$ denotes the $\mathbb{G}$-predictable projection of $\gamma$. In fact, we have for every bounded process $\zeta$

$$
\begin{aligned}
E\left[\int_{0}^{T} \zeta_{s} d\left(\int \gamma d B\right)_{s}^{p, G_{T}}\right] & =E\left[\int_{0}^{T}\left(\mathbb{G}_{s} \zeta_{s}\right) \gamma_{s} d B_{s}\right] \\
& =E\left[\int_{0}^{T}\left({ }^{p, G_{s}} \zeta_{s}\right) \gamma_{s} d B_{s}^{p, G_{t}}\right] \\
& =E\left[\int_{0}^{T} p, G_{r}\left(\left({ }^{p, G_{s}} \zeta_{s}\right) \gamma_{s}\right) d B_{s}\right] \\
& =E\left[\int_{0}^{T}\left(p, G_{s} \zeta_{s}\right)\left({ }^{p, G_{s}} \gamma_{s}\right) d B_{s}\right]
\end{aligned}
$$

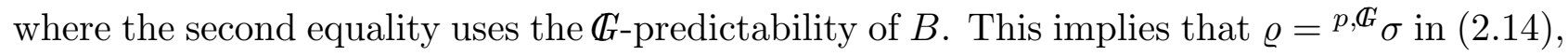
so (2.15) simplifies to

$$
\vartheta_{t}^{H}=\left({ }^{p, G_{t}} \sigma_{t}\right)^{\mathrm{inv}} p, G_{t}\left(\sigma_{t} \xi_{t}^{H}\right) .
$$


If, in addition, $\sigma$ is $\mathbb{G}$-predictable, i.e., if $\left\langle X^{i}, X^{j}\right\rangle$ is $\mathbb{G}$-predictable for all $i, j$, then (3.1) reduces to

$$
\vartheta_{t}^{H}={ }^{p, G_{t}} \xi_{t}^{H}
$$

In particular, this gives the solution to (2.3) or (2.11) if $X$ is $\mathbb{G}$-adapted, since $B$ and $\sigma$ can both be chosen $\mathbb{G}$-predictable in that case. Note that (3.2) then provides the connection between the Galtchouk-Kunita-Watanabe decompositions of $H$ with respect to $\mathbb{F}$ and $\mathbb{G}$, respectively.

\subsection{The one-dimensional case}

For $d=1$, the preceding formulae can be simplified even further. First of all, $\sigma$ and $\gamma$ commute for any $\gamma$ and so the proof of Theorem 2.5 shows that (2.15) can be written as

$$
\vartheta_{t}^{H}=\frac{d\left(\int \sigma \xi^{H} d B\right)_{t}^{p, G_{r}}}{d\left(\int \sigma d B\right)_{t}^{p, G r}}=\frac{d\left(\int \xi^{H} d\langle X\rangle\right)_{t}^{p, G_{r}}}{d\langle X\rangle_{t}^{p, G_{F}}} .
$$

We remark that a perfectly analogous result also holds for $d>1$ in the special case where the components of $X$ are pairwise strongly orthogonal; this is clear from (2.15) since $\sigma$ is then a diagonal matrix.

If, in addition, $B$ is now $\mathbb{G}$-predictable, (3.1) yields

$$
\vartheta_{t}^{H}=\frac{p, G_{t}\left(\sigma_{t} \xi_{t}^{H}\right)}{p, G_{T} \sigma_{t}}=\frac{E\left[\sigma_{t} \xi_{t}^{H} \mid \mathcal{G}_{t-}\right]}{E\left[\sigma_{t} \mid \mathcal{G}_{t-}\right]} .
$$

In particular, (3.3) holds if $\langle X\rangle$ is absolutely continuous with respect to Lebesgue measure since we can then choose $B_{t}:=t$. We could for instance think of a Black-Scholes type model with stochastic volatility where $X$ is given as the solution of the stochastic differential equation

$$
d X_{t}=v_{t-} X_{t} d W_{t}
$$

In that case, (3.3) takes the form

$$
\vartheta_{t}^{H}=\frac{E\left[\xi_{t}^{H} v_{t-}^{2} X_{t}^{2} \mid \mathcal{G}_{t-}\right]}{E\left[v_{t-}^{2} X_{t}^{2} \mid \mathcal{G}_{t-}\right]}
$$

this result was already obtained by Di Masi/Platen/Runggaldier (1993).

Finally we consider the case where $\langle X\rangle$ is $\mathbb{G}$-predictable. (2.17) then shows that $\vartheta^{H}$ is characterized by the property that

$$
0=E\left[\int_{0}^{T}\left(\xi_{u}^{H}-\vartheta_{u}^{H}\right) d X_{u} \int_{0}^{T} \gamma_{u} d X_{u}\right]=E\left[\int_{0}^{T} \xi_{u}^{H} \gamma_{u} d\langle X\rangle_{u}\right]-E\left[\int_{0}^{T} \vartheta_{u}^{H} \gamma_{u} d\langle X\rangle_{u}\right]
$$

for every $\gamma \in \Theta(\mathbb{G})$. If we denote by $P_{X}$ the measure $P \times\langle X\rangle$ on the product space $\Omega \times[0, T]$ with the $\sigma$-algebra $\mathcal{P}(\mathbb{G})$ of $\mathbb{G}$-predictable sets, (3.4) shows that $\vartheta^{H}$ is given by the conditional expectation

$$
\vartheta^{H}=E_{X}\left[\xi^{H} \mid \mathcal{P}(\mathbb{G})\right]
$$


i.e., by projecting $\xi^{H}$ on $\mathcal{P}(\mathbb{G})$.

\subsection{An example with delayed information}

As a first example, suppose that $X$ is given by the usual Black-Scholes model of geometric Brownian motion, but that information becomes available only with a certain delay. More precisely, let $X$ be the solution of

$$
d X_{t}=v X_{t} d W_{t}
$$

where $W$ is a Brownian motion, the volatility $v>0$ is for simplicity constant and $\mathbb{F}=\mathbb{F}^{W}$ is the augmentation of the filtration generated by $W$. To describe the delay in information, let $a:[0, T] \rightarrow[0, T]$ be an increasing RCLL function with $a(0)=0, a(T)=T$ and $a(t) \leq t$ for $t \in[0, T]$, and take

$$
\mathcal{G}_{t}:=\mathcal{G}_{t}^{\prime}:=\mathcal{F}_{a(t)} \quad, \quad 0 \leq t \leq T .
$$

More generally, we could of course take $\mathcal{G}_{t}^{\prime}:=\mathcal{F}_{a^{\prime}(t)}$ for another function $a^{\prime}(t)$ satisfying the same conditions as $a(t)$ and, in addition, $a(t) \leq a^{\prime}(t)$. If we choose $a(t)$ to be piecewise constant, this formulation includes in particular a model with discrete-time observations. Due to $a(T)=T$, condition (2.1) is satisfied, and so $\vartheta^{H}$ is given by

$$
\vartheta_{t}^{H}=\frac{E\left[\xi_{t}^{H} X_{t}^{2} \mid \mathcal{F}_{a(t)-}\right]}{E\left[X_{t}^{2} \mid \mathcal{F}_{a(t)-}\right]}
$$

thanks to (3.3).

To obtain more specific results, suppose now that $H$ has the form

$$
H=h\left(X_{T}\right)
$$

for a sufficiently nice function $h$. Denoting by $u(t, x)$ the solution of

$$
0=u_{t}+\frac{1}{2} v^{2} x^{2} u_{x x}
$$

with the boundary condition

$$
u(T, x)=h(x) \quad \text { for } x \geq 0,
$$

we then have the well-known expressions

$$
V_{t}^{H}=u\left(t, X_{t}\right)
$$

and

$$
\xi_{t}^{H}=u_{x}\left(t, X_{t}\right) \text {. }
$$

Inserting this into (3.5) and using the explicit expression for $X$ then leads to

$$
\vartheta_{t}^{H}=\left.E\left[u_{x}\left(t, y e^{Z}\right)\right]\right|_{y=X_{a(t)-}}
$$

with $Z \sim \mathcal{N}\left(\frac{3}{2} s^{2}, s^{2}\right)$ and $s^{2}=v^{2}(t-a(t)-)$. In the same way, we obtain

$$
\eta_{t}^{H}=\left.E\left[u\left(t, y e^{\widetilde{Z}}\right)-y u_{x}\left(t, y e^{\widehat{Z}}\right)\right]\right|_{y=X_{a(t)-}}
$$


with $\widetilde{Z} \sim \mathcal{N}\left(-\frac{1}{2} s^{2}, s^{2}\right)$ and $\widehat{Z} \sim \mathcal{N}\left(\frac{1}{2} s^{2}, s^{2}\right)$. For a specific choice of $h$, these expressions can be evaluated numerically.

As a concrete example illustrating the preceding computations, consider the claim

$$
H=\left(X_{T}-K\right)^{2}
$$

Then we get

$$
\begin{aligned}
\xi_{t}^{H} & =2\left(e^{v^{2}(T-t)} X_{t}-K\right), \\
V_{t}^{H}-\xi_{t}^{H} X_{t} & =K^{2}-e^{v^{2}(T-t)} X_{t}^{2}
\end{aligned}
$$

and

$$
\begin{aligned}
& \vartheta_{t}^{H}=2\left(e^{v^{2}(T-a(t)-)} X_{a(t)-} e^{v^{2}(t-a(t)-)}-K\right), \\
& \eta_{t}^{H}=K^{2}-e^{v^{2}(T-a(t)-)} X_{a(t)-}^{2} .
\end{aligned}
$$

Note that even for $a(t)$ piecewise constant, $\vartheta^{H}$ is in general not piecewise constant.

\subsection{A jump process example with discrete observations}

As a second example, we consider a two-sided jump process as in Föllmer/Sondermann (1986). Let $X$ be the solution of

$$
d X_{t}=\delta X_{t-}\left(d N_{t}^{+}-d N_{t}^{-}\right)
$$

where $N^{ \pm}$are independent Poisson processes with the same parameter $\lambda>0$ and $\delta>0$ is a fixed constant. The filtration $\mathbb{F}$ is the one generated by $N^{ \pm}$. The explicit solution of (3.6) is

$$
X_{t}=X_{0}(1+\delta)^{N_{t}^{+}}(1-\delta)^{N_{t}^{-}}
$$

For a contingent claim of the form $H=h\left(X_{T}\right)$, we thus obtain

$$
\begin{aligned}
V_{t}^{H} & =u\left(t, X_{t}\right), \\
\xi_{t}^{H} & =\frac{u\left(t,(1+\delta) X_{t-}\right)-u\left(t,(1-\delta) X_{t-}\right)}{2 \delta X_{t-}}=\frac{\left(\Delta^{+}-\Delta^{-}\right)\left(t, X_{t-}\right)}{2 \delta X_{t-}}
\end{aligned}
$$

with

$$
u(t, x):=\sum_{k, \ell=0}^{\infty} h\left(x(1+\delta)^{k}(1-\delta)^{\ell}\right) e^{-2 \lambda(T-t)} \frac{(\lambda(T-t))^{k+\ell}}{k ! \ell !}
$$

and

$$
\Delta^{ \pm}(t, x):=u(t,(1 \pm \delta) x)-u(t, x) .
$$

Note that (3.8) corrects an error in Example 2 of Föllmer/Sondermann (1986).

In order to model discrete-time observations, we now fix a partition $0=t_{0}<t_{1}<\ldots<$ $t_{n}=T$ of $[0, T]$ and take

$$
\mathcal{G}_{t}:=\sigma\left(X_{t_{k}} ; t_{k} \leq t\right)
$$


This means that we are only allowed to observe the price process $X$ at the discrete time instants $t_{k}$. Because of $t_{n}=T$, condition (2.1) is satisfied. Moreover, we have $\mathcal{G}_{t-}=\mathcal{G}_{t_{k}}$ for $t \in\left(t_{k}, t_{k+1}\right]$, and since

$$
\langle X\rangle_{t}=\int_{0}^{t} 2 \delta^{2} X_{s-}^{2} \lambda d s
$$

(3.7) yields

$$
\vartheta_{t}^{H}=\frac{E\left[\xi_{t}^{H} X_{t-}^{2} \mid \mathcal{G}_{t-}\right]}{E\left[X_{t-}^{2} \mid \mathcal{G}_{t-}\right]}=\sum_{k=0}^{n-1} \frac{E\left[\xi_{t}^{H} X_{t-}^{2} \mid \mathcal{G}_{t_{k}}\right]}{E\left[X_{t-}^{2} \mid \mathcal{G}_{t_{k}}\right]} I_{\left(t_{k}, t_{k+1}\right]}(t)
$$

By using the properties of the Poisson process, we can rewrite this expression as

$$
\begin{aligned}
\vartheta_{t}^{H}=\sum_{k=0}^{n-1} \frac{1}{2 \delta X_{t_{k}}}\left(E \left[u\left(t, y_{k}(1+\delta)^{Z_{k, t}^{+}+1}(1-\delta)^{Z_{k, t}^{-}}\right)\right.\right. \\
\left.\left.\quad-u\left(t, y_{k}(1+\delta)^{Z_{k, t}^{+}}(1-\delta)^{Z_{k, t}^{-}+1}\right)\right]\left.\right|_{y_{k}=X_{t_{k}}}\right) I_{\left.t_{k}, t_{k+1}\right]}(t),
\end{aligned}
$$

where the $Z_{k, t}^{ \pm}$are independent Poisson random variables with parameters $(1 \pm \delta) \lambda\left(t-t_{k}\right)$. In general, this expectation will have to be computed numerically.

For the special case where $H=X_{T}^{2},(3.7)$ and (3.9) can be explicitly calculated to give

$$
\xi_{t}^{H}=2 e^{2 \lambda \delta^{2}(T-t)} X_{t-}
$$

and

$$
\vartheta_{t}^{H}=2 e^{2 \lambda \delta^{2}\left(T-t_{k}\right)} X_{t_{k}} e^{2 \lambda \delta^{2}\left(t-t_{k}\right)} \quad \text { for } t \in\left(t_{k}, t_{k+1}\right] .
$$

Note that as in the preceding example, $\vartheta^{H}$ is again not constant on the partition intervals $\left(t_{k}, t_{k+1}\right]$.

\section{References}

C. Dellacherie and P.-A. Meyer (1982), "Probabilities and Potential B", North-Holland

G. B. Di Masi, E. Platen and W. J. Runggaldier (1993), "Hedging of Options under Discrete Observation on Assets with Stochastic Volatility", preprint, University of Padova

H. Föllmer and D. Sondermann (1986), "Hedging of Non-Redundant Contingent Claims", in: W. Hildenbrand and A. Mas-Colell (eds.), Contributions to Mathematical Economics, 205223

J. M. Harrison and S. R. Pliska (1981), "Martingales and Stochastic Integrals in the Theory of Continuous Trading", Stochastic Processes and their Applications 11, 215-260

M. Schweizer (1988), "Hedging of Options in a General Semimartingale Model", Diss. ETHZ No. 8615

C. Stricker (1990), "Arbitrage et Lois de Martingale", Annales de l'Institut Henri Poincaré 26, 451-460 